\title{
Article \\ Measuring technological change in primary sectors in EU-28 (2010-2015): extending the structural decomposition analysis
}

\author{
Xesús Pereira-López ${ }^{1 *}$, Małgorzata Anna Węgrzyńska ${ }^{2}$ and Napoleón Guillermo Sánchez-Chóez ${ }^{3,4}$ \\ 1 Department of Quantitative Economics and Institute of Development Studies of Galicia (IDEGA), Univer- \\ sity of Santiago de Compostela, Spain; xesus.pereira@usc.es \\ 2 Department of Finance and Accounting, Faculty of Economics, Poznań University of Life Sciences, Poland; \\ malgorzata.wegrzynska@up.poznan.pl \\ 3 Departamento de Estudios Organizacionales y Desarrollo Humano, Escuela Politécnica Nacional, Ecuador, \\ napoleon.sanchez@epn.edu.ec \\ 4 Institute of Development Studies of Galicia (IDEGA), University of Santiago de Compostela, Spain; napole- \\ onguillermo.sanchez@rai.usc.es \\ * Correspondence: xesus.pereira@usc.es
}

\begin{abstract}
This paper deals with the structural decomposition input-output for the economic analysis of agriculture, forestry and fishing in six EU-28 countries (Austria, Belgium, France, Germany, Italy and Spain) in the 2010-2015 period. The objective is to determine the causes of changes in production in these sectors with a particular focus on disaggregation technological change by distribution factors associated with a specific normalisation of the Leontief inverse. In calculating the net multipliers, an attempt was made to exclude sectors' own consumption appropriately. However, the treatment of own consumption upon introducing a time factor requires further investigation to avoid questionable quantifications. In general, typical characteristics of primary sectors include the accumulation of a significant amount of their own consumption, facilitated by symmetric accounting. Therefore, attention is drawn to these sectors so as to reveal possible analysis techniques that will provide nuance or validate existing techniques.
\end{abstract}

Keywords: structural decomposition analysis; technological change; primary sectors

\section{Introduction}

Structural Decomposition Analysis (SDA) is a practical tool that quantifies the causes of changes in a wide range of variables such as economic growth, energy consumption, labour demand, etc. Input-output (IO) SDA is usually defined as the study of economic change by comparing a series of static changes in the main variables of an IO model (Rose and Chen 1991).

The most traditional and straightforward SDA usually concerns three drivers of change: structural or technological changes relating to the effects caused by changes in the Leontief inverse, changes in final demand (which are usually divided into changes in levels and the composition of final demand), and the interaction or combination of both. There is an extensive related literature (see Hoekstra and Van Der Bergh 2003) using this methodology in various empirical studies to analyse production changes (Fujimagari 1989), changes in employment (Forssell 1990; Han 1995) or labour productivity . However, it is chiefly applied to energy consumption, $\mathrm{CO}_{2}$ emissions, and other environmental issues (Casler and Rose 1998; Chen and Wu 1994; Han and Lakshmanan 1994; Jacobsen 2000; Mukhopadhyay and Chakraborty 1999). There have also been numerous developmental proposals for the theoretical approach to the model (Chen and Wu 2008; E. Dietzenbacher and Los 1998; Rose and Casler 1996; Sonis et al. 1996; Wang et al. 2014). In other words, SDA is an appropriate methodology for analysing the driving forces behind changes in economic, energy, and environmental indicators. 
In general, SDA formulas have been modified by new ways of factor decomposition. However, changes in the Leontief inverse remain unnoticed. Therefore, it is proposed to decompose structural change by the effect (as a whole) caused by sectors' own consumption. Thus, a specific normalisation criterion by rows and columns of the model is applied.

In the field of agricultural operations, SDA is also used, especially with regard to environmental issues. Studies are available at EU level, for example, in Italy in 1999-2006, the analysis was applied to energy and environmental products to determine the impact of energy intensity and emissions, the Leontief effect and the final demand effect on changes in certain volumes, concluding that 'agriculture, hunting and forestry' are among the main sectors affecting emissions to the atmosphere (Cellura et al. 2012). The analysis was used to see how changes in final demand and technology affected water use changes for different crops in the EU. The results revealed that major crop producers experienced an increase in water use, mainly due to technology changes (Gerveni et al. 2020). Internal causes of the increase in global consumption-based GHG emissions (1995-2009) were also analysed, with agriculture playing an important role in developing countries (Liu et al. 2019). SDA was also applied to examine water use changes in agricultural production in the United States (1995-2010). It revealed an overall decrease in water use in the production of all crops due to more efficient irrigation systems (Avelino and Dall'erba 2020). However, there is no doubt that the largest number of conducted SDAs focused on China, both nationwide and at the provincial level. This method was used to determine drivers of metal consumption in China (1997-2015) in agriculture, industry, construction, and services (Song et al. 2019), and to estimate energy-related $\mathrm{CO}_{2}$ emissions and structural emissions reductions in Chinese agriculture (2007-2017). It was found, among other things, that increasing agricultural demand across the industrial system fostered the growth of these emissions ( $\mathrm{Yu}$ et al. 2020). Other similar provincial-level papers concerning China and using SDA, sometimes in combination with complementary techniques, include the works of Cai et al. (2019); Fang and Yang (2021); Feng et al. (2017); D. Li et al. (2020); W. Li et al. (2020); X. Li et al. (2020); L.-J. Liu and Liang (2017); Qian et al. (2018); Supasa et al. (2017); X. Wang et al. (2016); Zhi et al. (2014) and Xu et al. (2017). Studies of a socio-economic nature are uncommon, although they do exist. In China, for example, the sources of employment growth were analysed using this method. It was found that the contribution of exports to job creation increased significantly, especially in agriculture (Doan and Long 2019).

Empirically, given changes (in terms of output) in the primary sectors of the six countries of EU-28 between 2010 and 2015 are analysed based on symmetric IO tables (total flows). A relatively short period was chosen that was characterised by stagnation and even regression of macro-economic indicators. Therefore, it is expected that these sectors did not experience significant changes in their production structure. At the same time, it can be seen that price changes during this period were not significant, yet deflation based on 2010 was applied to the flows. The change in output is largely driven by final demand behaviour and can be broken down by its components. It is now necessary to see to what extent the technological change (the second cause of change) can be broken down according to the IO logic. The total effects are calculated based on the effect of final demand and direct and indirect requirement effects. This idea is embodied in the Leontief inverse construction, so it is amenable to decomposition by distribution coefficients (associated with a particular normalisation) corresponding to this logic.

In calculating net IO multipliers, the goal is to exclude sectors' own consumption from the calculation properly. Primary sectors tend to accumulate a large amount of own consumption in their cost structures, and the very development of symmetric accounting favours this situation. While extrapolating this recommended purification of multipliers to the notion of technological change, attention should be focused on these sectors to reveal possible analytical ways to nuance or enhance existing ones.

\section{Preliminary DATA}


The information provided by the IO tables is essential to highlight the different sources of change in output, in particular the information available in the intermediate input matrices, given that their structures are the primary reference in SDA. Indeed, they were extracted from a database (Eurostat 2015a) containing symmetric matrices in $64 \times 64$ basic prices of products for six EU-28 countries (Austria, Belgium, France, Germany, Italy and Spain), in 2010-2015. At the same time, the corresponding price deflation from the 2015 tables based on 2010 was performed. In this context, it should be noted that Eurostat has detailed information on deflation indicators (with 2010 as reference) of value added by sector (Eurostat 2015b). An analogous indicator by intermediate inputs is only available for Germany. Therefore, this approach was chosen because it includes the same criterion for the six countries analysed.

With respect to indirect inputs in the three economic activities included in primary sectors, Figure 1 shows that they did not change much between 2010 and 2015, and neither did the price index (it is given as an average). Crop and animal production, hunting and related service activities have a higher relative weight of intermediate inputs than the other two in these six countries. Logically, countries with access to the sea also excel in fishing and aquaculture. Thus, Figure 1 requires no further explanation.


Figure 1. Change in intermediate inputs in primary sectors and price index, 2010-2015.

Figure 2 shows the changes -2010 with an index of 100-in both value added and total output for the three aggregate sectors over the period analysed, indirectly subtracting changes in intermediate inputs. Four countries (Austria, Belgium, France and Spain) show 
very similar behaviour in these two variables (also including deflation with reference to 2010). Using value-added price indices as a criterion may be appropriate for calculating deflation of IO tables.

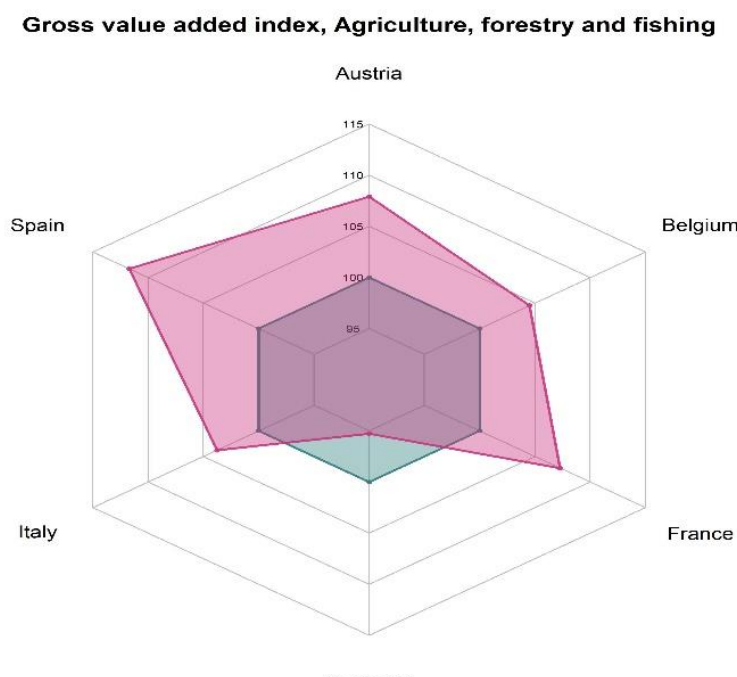

Germany

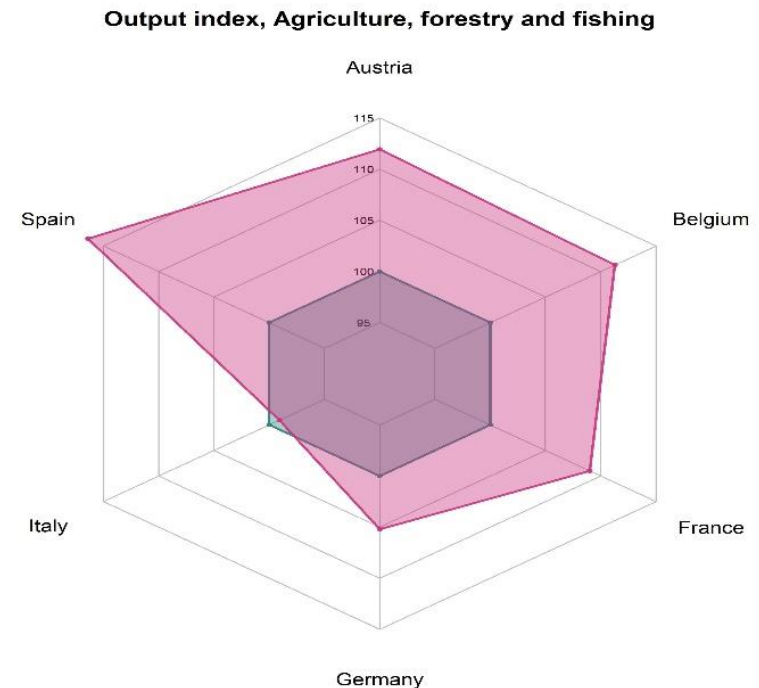

-2010
$\rightarrow 2015$

Figure 2. Change in value added and output of indicators, 2010-2015.

On the other hand, Figure 3 shows the percentage structure of final demand in the six countries studied (2015, including deflation with reference to 2010). The disaggregation into three elements, although one could go further (in it disaggregation). The importance of final consumption expenditure stands out in almost all cases. However, fishing exports have importance, especially in France and Germany. Table 1 presents the same information, but in value terms. The 2010 structure is very similar.

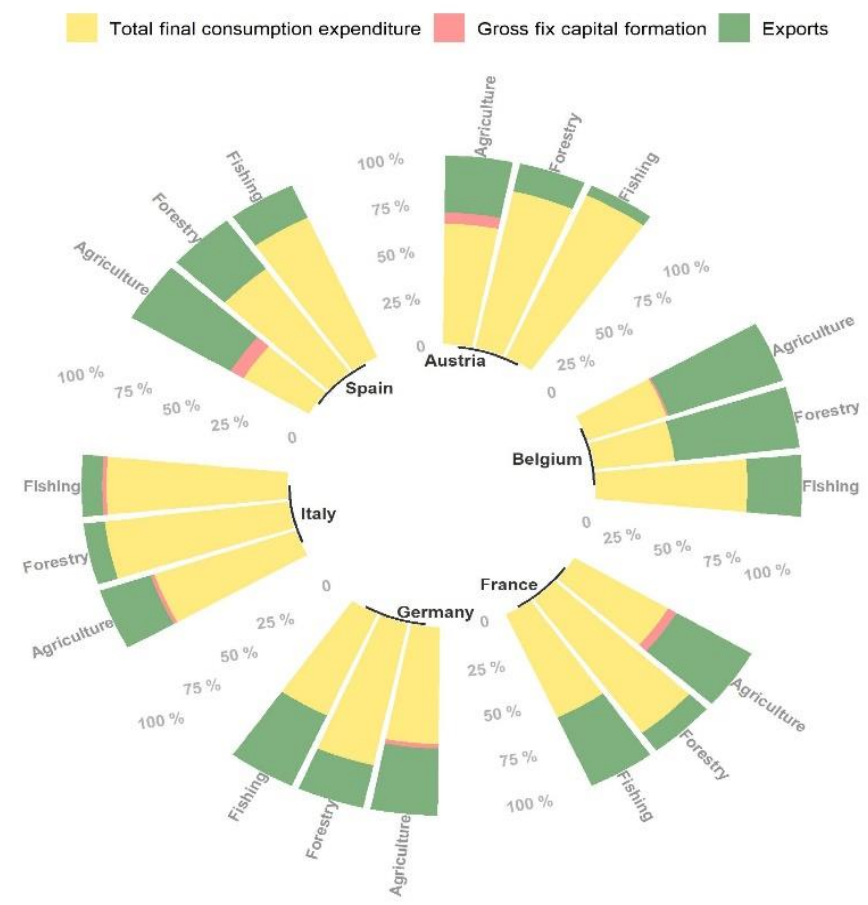

Figure 3. Final demand components by country (\%) in 2015.

Table 1. Final demand components by country (million EUR) in 2015. 


\begin{tabular}{ccccr}
\hline Country & Product & $\begin{array}{c}\text { Total final consump- } \\
\text { tion expenditure }\end{array}$ & $\begin{array}{c}\text { Gross fix capi- } \\
\text { tal formation }\end{array}$ & Exports \\
\hline \multirow{3}{*}{ Austria } & Agriculture & 2073.76 & 191.14 & 979.25 \\
& Forestry & 406.22 & 0.00 & 72.41 \\
& Fishing & 41.87 & 0.00 & 2.57 \\
& Agriculture & 2537.83 & 63.39 & 3701.04 \\
Belgium & Forestry & 93.40 & 0.00 & 145.75 \\
& Fishing & 167.03 & 0.00 & 60.04 \\
& Agriculture & 15015.05 & 1374.04 & 12086.93 \\
France & Forestry & 1154.17 & 0.00 & 152.98 \\
& Fishing & 783.25 & 0.00 & 519.02 \\
& Agriculture & 17318.48 & 674.49 & 9924.52 \\
Germany & Forestry & 1117.48 & 0.00 & 337.28 \\
& Fishing & 340.93 & 0.00 & 237.43 \\
& Agriculture & 13704.94 & 365.38 & 4897.40 \\
Italy & Forestry & 826.99 & 0.00 & 94.03 \\
& Fishing & 2066.41 & 47.70 & 236.75 \\
& Agriculture & 8260.62 & 1649.21 & 12520.33 \\
Spain & Forestry & 320.49 & 0.21 & 149.85 \\
& Fishing & 1942.74 & 0.00 & 448.47 \\
\hline
\end{tabular}

\section{Methodology}

SDA and its extensions have identified various sources of change in both the economy and its production sectors. To achieve the disaggregation of technological change, a specific modification of the SDA is required. Decompositions of the Leontief inverse vary, but the point is to look for the right one to achieve the stated goal.

\subsection{Normalisation of the Leontief Inverse Matrix}

The open demand model, or Leontief model, can be expressed as

$$
x=(I-A)^{-1} y=C y,
$$

where $x$ is the output vector, $(I-A)^{-1}$ is the Leontief inverse matrix, which is further simplified by $C$, and $y$ is the net import final demand vector. Here, $A$ is a matrix of technical coefficients (total flows). Its generic element, denoted by $a_{i j}$ represents the contribution of sector $i$ per unit of output of sector $j$. Furthermore, it is believed that the examined economy is broken down into $\mathrm{n}$ sectors.

The following is probably the most traditional way to present the Leontief inverse matrix

$$
C=I+A+A^{2}+\cdots
$$

where the generic element $\alpha_{i j}(i, j=1, \ldots, n)$ is presented as follows

$$
\left\{\begin{array}{c}
1+a_{i j}+\sum_{k=1}^{n} a_{i k} a_{k j}+\sum_{k_{1}=1}^{n} \sum_{k_{2}=1}^{n} a_{i k_{1}} a_{k_{1} k_{2}} a_{k_{2} j}+\cdots,(i=j) \\
a_{i j}+\sum_{k=1}^{n} a_{i k} a_{k j}+\sum_{k_{1}=1}^{n} \sum_{k_{2}=1}^{n} a_{i k_{1}} a_{k_{1} k_{2}} a_{k_{2} j}+\cdots,(i \neq j)
\end{array}\right.
$$

To search for alternative $C$, distributions, a diagonal matrix $\hat{D}$ is constructed with the elements of the main diagonal $C$ being the sum of the elements of the main diagonals of these $I, A, A^{2}, \cdots, A^{n}, \cdots$ matrices. In analytical terms, such a diagonal matrix can be expressed as follows: 


$$
\hat{D}=I+\operatorname{diag}(E)+\operatorname{diag}\left(E^{2}\right)+\cdots+\operatorname{diag}\left(E^{n}\right)+\cdots,
$$

where the generic element is presented as follows:

$$
d_{i}=\alpha_{i i}=1+a_{i i}+\sum_{k=1}^{n} a_{i k} a_{k i}+\sum_{k_{1}=1}^{n} \sum_{k_{2}=1}^{n} a_{i k_{1}} a_{k_{1} k_{2}} a_{k_{2} i}+\cdots
$$

From here one can derive the decomposition of the Leontief inverse matrix:

$$
\begin{aligned}
C & =\hat{D} \hat{D}^{-1} C=\left(I+\operatorname{diag}(E)+\operatorname{diag}\left(E^{2}\right)+\cdots+\operatorname{diag}\left(E^{n}\right)+\cdots\right) \hat{D}^{-1} C= \\
& =\hat{D}^{-1} C+\operatorname{diag}(E) \hat{D}^{-1} C+\operatorname{diag}\left(E^{2}\right) \hat{D}^{-1} C+\cdots+\operatorname{diag}\left(E^{n}\right) \hat{D}^{-1} C+\cdots
\end{aligned}
$$

In this decomposition, normalisation is indicated by the rows of the $C$ matrix,

$$
\tilde{C}^{f}(\text { row })=\hat{D}^{-1} C=\left(\begin{array}{cccc}
1 & \frac{\alpha_{12}}{\alpha_{11}} & \cdots & \frac{\alpha_{1 n}}{\alpha_{11}} \\
\frac{\alpha_{21}}{\alpha_{22}} & 1 & \cdots & \frac{\alpha_{2 n}}{\alpha_{22}} \\
\vdots & \vdots & \ddots & \vdots \\
\frac{\alpha_{n 1}}{\alpha_{n n}} & \frac{\alpha_{n 2}}{\alpha_{n n}} & \cdots & 1
\end{array}\right) .
$$

This normalisation criterion was applied, albeit partially (Jeong 1982, 1984), to the direct and indirect input demand matrix and $(C-I)$, was then Gim and Kim (1998) used for this matrix in its entirety. In this way, the $C$ elements are adjusted by row according to the value of the main diagonal (associated with the production branch); that is, the elements are amenable to be used for the quantification of net multipliers and combine from a methodological point of view with the hypothetical extraction method.

Another normalisation of $C$ is given by

$$
\tilde{C}^{g}(\text { row })=\left(I-\hat{D}^{-1}\right) C=\left(\begin{array}{cccc}
\alpha_{11}-1 & \frac{\alpha_{11}-1}{\alpha_{11}} \alpha_{12} & \cdots & \frac{\alpha_{11}-1}{\alpha_{11}} \alpha_{1 n} \\
\frac{\alpha_{22}-1}{\alpha_{22}} \alpha_{21} & \alpha_{22}-1 & \cdots & \frac{\alpha_{22}-1}{\alpha_{22}} \alpha_{2 n} \\
\vdots & \vdots & \ddots & \vdots \\
\frac{\alpha_{n n}-1}{\alpha_{n n}} \alpha_{n 1} & \frac{\alpha_{n n}-1}{\alpha_{n n}} \alpha_{n 2} & \cdots & \alpha_{n n}-1
\end{array}\right) .
$$

This matrix is constructed in a similar way as (7), except that the transformations are conditioned on the effects provided by $\left(\alpha_{i i}-1\right), i=1,2 \ldots, n$; i.e. the direct effect of final demand on the multipliers is excluded.

Next, one can see the role that normalisation by $C$ rows plays in the model itself. Thus, if one considers (1) and makes some modification, the following distribution is found

$$
\begin{aligned}
x & =C y=I C y=\left(\hat{D}^{-1}+I-\hat{D}^{-1}\right) C y= \\
& =\left[\hat{D}^{-1} C+\left(I-\hat{D}^{-1}\right) C\right] y=\hat{D}^{-1} C y+\left(I-\hat{D}^{-1}\right) C y,
\end{aligned}
$$

in a nutshell, it can be seen that

$$
x=\tilde{C}^{f}(\text { row }) y+\tilde{C}^{g}(\text { row }) y .
$$


Therefore, the generic element $C$ is broken down by two (or more) distribution factors as follows:

$$
\alpha_{i j}=\frac{1}{\alpha_{i i}} \alpha_{i j}+\frac{\alpha_{i i}-1}{\alpha_{i i}} \alpha_{i j}
$$

Disaggregation $\alpha_{i j}$ down based on the effect given by an increase in one unit of final demand and the other effects (given at different stages of production) that make up the $\alpha_{i i}$ normalising element. Thus, a production sector with a high own consumption will prefer normalising elements with a higher value. As a result, the distribution coefficient $\frac{1}{\alpha_{i i}}$ decreases and (simultaneously) the $\frac{\alpha_{i i}-1}{\alpha_{i i}}$ coefficient increases. The opposite is true in sectors with a low own consumption. This means that the calculation of net multipliers varies by sector. Of course, the $\frac{\alpha_{i i}-1}{\alpha_{i i}}$ factor can be further broken down according to (5), at least by distinguishing between direct and indirect requirements: $\frac{a_{i i}}{\alpha_{i i}}$ and $\frac{\alpha_{i i}-\left(1+a_{i i}\right)}{\alpha_{i i}}$.

The first equation of system (10) can be considered based on the following example

$$
x_{1}=\left[y_{1}+\frac{\alpha_{12}}{\alpha_{11}} y_{2}+\ldots+\frac{\alpha_{1 n}}{\alpha_{11}} y_{n}\right]+\left[\left(\alpha_{11}-1\right) y_{1}+\frac{\alpha_{11}-1}{\alpha_{11}} \alpha_{12} y_{2}+\ldots \frac{\alpha_{11}-1}{\alpha_{11}} \alpha_{1 n} y_{n}\right] .
$$

Another possible transformation of $C$ is as follows:

$$
\begin{aligned}
C & =C \hat{D} \hat{D}^{-1}=C\left(I+\operatorname{diag}(E)+\operatorname{diag}\left(E^{2}\right)+\cdots+\operatorname{diag}\left(E^{n}\right)+\cdots\right) \hat{D}^{-1}= \\
& =C \hat{D}^{-1}+C \operatorname{diag}(E) \hat{D}^{-1}+C \operatorname{diag}\left(E^{2}\right) \hat{D}^{-1}+\cdots+C \operatorname{diag}\left(E^{n}\right) \hat{D}^{-1}+\cdots
\end{aligned}
$$

Therefore, normalisation by columns in the Leontief inverse matrix is found analogously

$$
\tilde{C}^{f}(\mathrm{col})=C \hat{D}^{-1}
$$

and

$$
\tilde{C}^{g}(\mathrm{col})=C\left(I-\hat{D}^{-1}\right) .
$$

This normalisation was applied to $(C-I)$, and Gim and Kim (2005), Miller and Blair (2009) applied it directly to $C$.

Similarly, one can see the role of normalisation by columns in the following model

$$
\begin{aligned}
x & =C y=C I y=C\left(\hat{D}^{-1}+I-\hat{D}^{-1}\right) y= \\
& =C \hat{D}^{-1} y+C\left(I-\hat{D}^{-1}\right) y=\tilde{C}^{f}(\mathrm{col}) y+\tilde{C}^{g}(\mathrm{col}) y .
\end{aligned}
$$

It yields two alternative distributions for the Leontief inverse matrix. And thus

$$
C=\tilde{C}^{f}(\text { row })+\tilde{C}^{g}(r o w)=\tilde{C}^{f}(\mathrm{col})+\tilde{C}^{g}(\mathrm{col}) .
$$

From a mathematical point of view, one can resort to a combination of two matrix normalisation methods

$$
2 x=\left[\tilde{C}^{f}(\text { row }) y+\tilde{C}^{g}(\text { row }) y\right]+\left[\tilde{C}^{f}(\mathrm{col}) y+\tilde{C}^{g}(\mathrm{col}) y\right] .
$$


To conclude

$$
x=0.5\left[\tilde{C}^{f}(\text { row })+\tilde{C}^{f}(\mathrm{col})\right] y+0.5\left[\tilde{C}^{g}(\text { row })+\tilde{C}^{g}(\mathrm{col})\right] y,
$$

which as a shortened demand model can be expressed as

$$
x=\tilde{C}^{f} y+\tilde{C}^{g} y .
$$

Thus, the demand model -expressed in this way- breaks the overall impact into two factors; it determines the sector's importance in terms of its relative external contribution to market interdependence, and it reproduces the size of the corresponding economy.

\subsection{Structural decomposition Analysis}

The purpose of structural decomposition analysis is to isolate the main sources of change in the economy and its production sectors over a certain period. More specifically, it is a matter of detecting what part of the economic change between two points in time is related to technological change factors or productive efficiency, and what part is due to changes in final demand.

Thus, the increase in output between year 0 and year 1 is

$$
\Delta x=C_{1} y_{1}-C_{0} y_{0}
$$

because $y_{1}=y_{0}+\Delta y$ and $C_{1}=C_{0}+\Delta C$, it is possible to express the above in different ways.

It should be noted that

$$
\Delta x=C_{1}\left(y_{0}+\Delta y\right)-\left(C_{1}-\Delta C\right) y_{0}=\Delta C y_{0}+C_{1} \Delta y,
$$

or alternatively,

$$
\Delta x=\left(C_{0}+\Delta C\right) y_{1}-C_{0}\left(y_{1}-\Delta y\right)=\Delta C y_{1}+C_{0} \Delta y,
$$

or even,

$$
\Delta x=\Delta C y_{0}+C_{0} \Delta y+\Delta C(\Delta y)
$$

It also follows that

$$
\Delta x=\Delta C y_{1}+C_{1} \Delta y-\Delta C(\Delta y)
$$

And (24) and (25) give the average of

$$
\Delta x=\frac{1}{2}\left[\Delta C\left(y_{0}+y_{1}\right)+\left(C_{0}+C_{1}\right) \Delta y\right]=\Delta C \bar{y}+\bar{C} \Delta y,
$$

where the vector $\bar{y}=0.5\left(y_{0}+y_{1}\right)$ and matrix $\bar{C}=0.5\left(C_{0}+C_{1}\right)$.

To interpret a technological change in economic sectors more precisely, a methodological proposal is presented that uses a specific normalisation criterion - by row and column - of the Leontief inverse matrix. In fact, it is possible to find an alternative (additive) distribution. Therefore, decomposing the inverse in (21) yields the following

$$
\Delta x=\left[\tilde{C}_{1}^{g}+\tilde{C}_{1}^{f}\right] y_{1}-\left[\tilde{C}_{0}^{g}+\tilde{C}_{0}^{f}\right] y_{0} .
$$

By performing the following operation

$$
\Delta x=\tilde{C}_{1}^{g} y_{1}-\tilde{C}_{0}^{g} y_{0}+\tilde{C}_{1}^{f} y_{1}-\tilde{C}_{0}^{f} y_{0},
$$

because $y_{1}=y_{0}+\Delta y$ the following substitutions can be made 


$$
\begin{aligned}
\Delta x & =\tilde{C}_{1}^{g}\left[y_{0}+\Delta y\right]-\tilde{C}_{0}^{g} y_{0}+\tilde{C}_{1}^{f}\left[y_{0}+\Delta y\right]-\tilde{C}_{0}^{f} y_{0}= \\
& =\tilde{C}_{1}^{g} y_{0}+\tilde{C}_{1}^{g} \Delta y-\tilde{C}_{0}^{g} y_{0}+\tilde{C}_{1}^{f} y_{0}+\tilde{C}_{1}^{f} \Delta y-\tilde{C}_{0}^{f} y_{0}= \\
& =\Delta \tilde{C}^{g} y_{0}+\Delta \tilde{C}^{f} y_{0}+C_{1} \Delta y .
\end{aligned}
$$

On the other hand, because $y_{0}=y_{1}-\Delta y$, another alternative decomposition is found

$$
\Delta x=\Delta \tilde{C}^{g} y_{1}+\Delta \tilde{C}^{f} y_{1}+C_{0} \Delta y .
$$

This produces an additive expression of the modified SDA

$$
\Delta x=\frac{1}{2}\left[\Delta \tilde{C}^{g}+\Delta \tilde{C}^{f}\right]\left(y_{0}+y_{1}\right)+\frac{1}{2}\left(C_{0}+C_{1}\right) \Delta y,
$$

or shortened as follows:

$$
\Delta x=\left[\Delta \tilde{C}^{f} \bar{y}+\Delta \tilde{C}^{g} \bar{y}\right]+\bar{C} \Delta y .
$$

The change vector $\Delta \tilde{C}^{g} \bar{y}$ can be broken down by production stage, at the very least based on direct and indirect requirements.

\section{Results and discussion}

The proposed decomposition applies to the six EU-28 countries, in 2010-2015, for which we have homogeneous information based on symmetric IO tables of total flows. Agriculture is the most important of the three selected sectors and is, in fact, at the centre of attention. It was decided to present results concerning the other two similar sectors to test the extended methodology, although calculations were made for all production sectors.


Figure 4. Structure of main diagonal multipliers, 2010-2015.

The reference data are characterised by a slowdown -compared to previous yearsfor both final demand and output by sector. This circumstance is considered ideal to qualify the measurement of technological change using SDA. There has been an upward trend 
in these macro-economic indicators in the past, so there is no suspicion of possible overestimation in this area.

According to formula (5), the elements of the main diagonal of the Leontief inverse matrix can be divided into three effects - determined by final demand, direct requirement (own consumption) and indirect requirement. Figure 4 shows the disaggregation of the products examined. This information will be critical for interpreting the normalisation results described in the methodology section.

There are no significant differences between the two survey years, but there are some differences among countries. In fact, high values of these multipliers lead to sharp adjustments in the corresponding rows and/or columns, as will be shown in the normalised matrices, $\tilde{C}^{f}$ (row) and $\tilde{C}^{f}$ (col). For example, forestry's own consumption in Austria, France and Germany is very high and has its indirect effects, as can be seen in the figure. Moreover, in the case of Belgium and Italy, this sector's own consumption is zero. Thus, normalisation by row and/or column does not affect the Leontief inverse matrix.

Table 2. The disaggregation of changes in production by country (million EUR) from 2010 to 2015.

\begin{tabular}{|c|c|c|c|c|c|c|c|}
\hline \multirow[b]{2}{*}{ Country } & \multirow[b]{2}{*}{ Product } & \multicolumn{2}{|c|}{ Output change } & \multicolumn{3}{|c|}{ Technological change } & \multirow{2}{*}{$\begin{array}{c}\text { Final demand } \\
\text { change }\end{array}$} \\
\hline & & Value & $\%$ & $\begin{array}{l}\text { Standardised } \\
\text { by } \\
\text { Final demand }\end{array}$ & $\begin{array}{l}\text { Standardised } \\
\text { by direct re- } \\
\text { quirements }\end{array}$ & $\begin{array}{l}\text { Standard- } \\
\text { ised by indi- } \\
\text { rect require- } \\
\text { ments }\end{array}$ & \\
\hline \multirow{3}{*}{ Austria } & Agriculture & 999.25 & 18.59 & 544.45 & 81.78 & 44.81 & 328.21 \\
\hline & Forestry & 52.46 & 2.41 & -64.97 & -20.66 & -25.63 & 163.71 \\
\hline & Fishing & 0.64 & 1.52 & 10.52 & -0.32 & -0.03 & -9.53 \\
\hline \multirow{3}{*}{ Belgium } & Agriculture & 1042.71 & 12.34 & 1548.82 & 310.86 & 318.44 & -1135.41 \\
\hline & Forestry & -6.35 & -1.57 & -25.13 & -3.27 & 0.07 & 21.98 \\
\hline & Fishing & -33.23 & -25.64 & -63.42 & -3.96 & -2.74 & 36.88 \\
\hline \multirow{3}{*}{ France } & Agriculture & 4479.31 & 7.12 & 1568.98 & 418.71 & 241.55 & 2250.06 \\
\hline & Forestry & 152.84 & 3.06 & -710.22 & -163.50 & -87.95 & 1114.52 \\
\hline & Fishing & 122.14 & 5.85 & 178.06 & 8.74 & 4.40 & -69.06 \\
\hline \multirow{3}{*}{ Germany } & Agriculture & 5763.43 & 14.14 & 4860.93 & 599.65 & 365.42 & -62.57 \\
\hline & Forestry & 1085.35 & 34.35 & -29.51 & -85.93 & -49.97 & 1250.75 \\
\hline & Fishing & 44.10 & 11.67 & 124.67 & 12.58 & 3.44 & -96.59 \\
\hline \multirow{3}{*}{ Italy } & Agriculture & -1763.27 & -3.82 & 7.15 & -35.33 & -8.64 & -1726.45 \\
\hline & Forestry & 89.72 & 7.06 & 145.97 & 8.90 & 3.37 & -68.51 \\
\hline & Fishing & -180.33 & -8.33 & 227.49 & 19.53 & 3.14 & -430.49 \\
\hline \multirow{3}{*}{ Spain } & Agriculture & 5512.51 & 14.00 & 1755.95 & 903.09 & 394.84 & 2458.64 \\
\hline & Forestry & 233.58 & 19.45 & 159.77 & 51.30 & 14.65 & 7.86 \\
\hline & Fishing & -345.30 & -14.99 & -198.02 & -18.62 & -2.75 & -125.91 \\
\hline
\end{tabular}

Table 2 shows the results concerning the three primary sectors from 2010 to 2015 . Although for computational purposes, all production sectors were considered. The impact of the final demand vector is not broken down. However, many possible disaggregation could arise with respect to this vector, so it would be possible to refer to extended versions of this formula. Also, instead of working with an additive format, one can use a multiplicative format. The various disaggregation possibilities shown in Table 2 do not present a common pattern, although they do show some commonalities that are described below. With respect to agriculture, there is increasing variability in production, except for Italy, which can be explained by technological change and final demand changes. However, these recent changes do not always follow the same path. Overall, changes in the other 
sectors, i.e. forestry and fishing, are not as significant. However, they show differences other than in the case of agriculture.

The focus is put on variation due to technological change. The variation is explained by positive or negative changes in inverses and the mean vector of final demand, which is always positive. This means that the sign will be determined by changes in Leontief inverse matrices. In this sense, it should be noted that reduction in the share of intermediate demand results in an increase in the final demand share. Therefore, for the purposes of the inverse, it implies lower values of its components. Over time, this means that negative changes in the inverse imply that there is less dependence on intermediate inputs. In a period such as the one under consideration, which is characterised by an economic crisis and simoultaneously covers only a few years, it can be assumed that almost no changes have taken place in its production structures. Therefore, such improvement of the overall figures concerning technological change by rough subtracting the component of assigned sectoral own consumption could be an adequate alternative for an analysis.

Figure 5 confirms a table of results, but focuses only on the decomposition of technological changes. A decomposition of the change indicated by final demand can also be performed, however, that is beyond the scope of this article. The results were shown as percentages to facilitate their visual comparison. Again, heterogeneous behaviour is evident. It is clear that the disaggregation of elements of the main diagonal presented in Figure 4 determines these results because those elements are used as distribution factors. For example, it is clear that agriculture in Italy shows technological improvement, although when we go into detail about it, the change in own consumption determines this global figure and the other two components end up compensating because they have a different sign. Another example to consider is Forestry in Germany, with high own consumption, as stated above, where the factor assigned to it explains much of the reduction in output in this way - if this component is subtracted, the technological change will not be so clear.
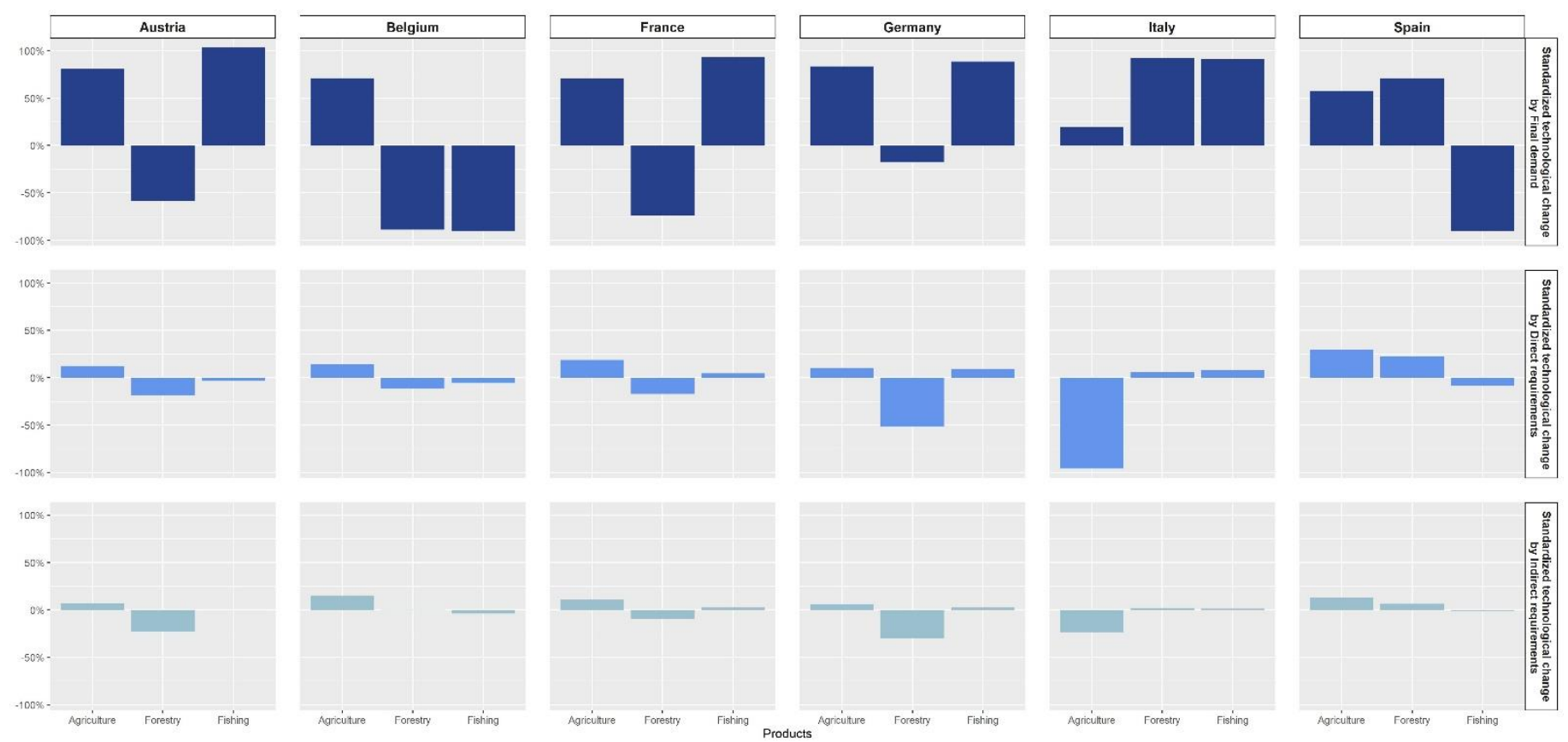

Figure 5. The disaggregation of technological changes by country from 2010 to 2015.

The value of the main diagonal's elements (Leontief inverse matrix) depends largely on own consumption, besides the interrelationships of each production branch with the others that make up the economy. Even mere errors in calculation in terms of own consumption may be considered to be technological changes as they are not. In this sense, the products of agriculture vary and they are presented in a very aggregated form in terms of the formatting processes of symmetric IO tables. Moreover, the symmetric format almost 
always needs to be adjusted by algorithms that easily tend to raise figures. In future research, the output changes could be analysed using rectangular IO models because origindestination tables present a larger disaggregation of basic products and do not depend on adjustments that are purely mathematical and ensure balance sheet equilibrium. However, in some way they may distort true relationships between product demand and output due to heterogeneous industrial sectors.

\section{Conclusions}

Primary sectors are adequate for testing the extended SDA. These are sectors that are characterised by maintaining strong links with the rest of the economy, yet they tend to collect the larger-than-average data concerning own consumption at the same time. Compared to the Leonteif model, own consumption - through normalisation - does not change the accounting balance, hence it is possible to make transformations of the above-mentioned model, which facilitate the calculation of net effects. Furthermore, the presented normalisations of the Leontief inverse matrix and its decomposition facilitate the calculation of the importance of a sector in terms of its external contribution -associated with sectoral interdependencies- and also reproduce the size of the corresponding economy. In terms of comparing the accounting frameworks, whether for the economy as a whole or for the manufacturing sector, the sectoral own consumption can also be relativised. In this way, it can be concluded, at least in part, what effect they have on the calculation of multipliers to compare inverses from different years. The sensitiveness of inverse elements allows nuance or other means that adjust or refine concepts such as technological change. In the processes of constructing homogeneous branches (a product equals to a branch) in equilibrium, some adjustments are applied to raise the figures to a higher value, whereby own consumption is an excellent candidate for the accumulation of errors (adjustments). Hence, the output changes can be analysed using rectangular IO models because they use a larger disaggregation of basic products and they are not subject to accounting adjustments.

Supplementary Materials: Not applicable.

Author Contributions: All authors have made substantial contributions. All authors read and approved the final manuscript.

\section{Funding:}

This research was funded by Faculty of Economics, Poznań University of Life Sciences, Poland.

Data Availability Statement: Data related to symmetric matrices in $64 \times 64$ basic prices of products for EU-28 are freely available in Eurostat's database at https:/ec.europa.eu/eurostat/web/esa-supply-use-input-tables/data/database. At the same time, data related to information on deflation indicators (with 2010 as reference) of value added by sector were obtained from the Eurostat https://appsso.eurostat.ec.europa.eu/nui/show.do?dataset=nama 10 a64\&lang=en.

Conflicts of Interest: The authors declare no conflict of interest.

\section{References}

Avelino, Andre F T., and Sandy Dall'erba. 2020. What factors drive the changes in water withdrawals in the U.S. Agriculture and food manufacturing industries between 1995 and 2010? Environmental Science and Technology 54: 10421-10434.

Cai, Beiming, Wei Zhang, Klaus Hubacek, Kuishuang Feng, Zhenliang Li, Yawen Liu, and Yu Liu. 2019. Drivers of virtual water flows on regional water scarcity in China. Journal of Cleaner Production 207: 1112-1122.

Casler, Stephen D., and Adam Rose. 1998. Carbon dioxide emissions in the U.S. economy: a structural decomposition analysis. Environmental and Resource Economics 11: 349-363.

Cellura, Maurizio, Sonia Longo, and Marina Mistretta. 2012. Application of the Structural Decomposition Analysis to assess the indirect energy consumption and air emission changes related to Italian households consumption. Renewable and Sustainable 
Energy Reviews 16: 1135-1145.

Chen, Chia Yon, and Rong Hwa Wu. 1994. Sources of change in industrial electricity use in the Taiwan economy, 1976-1986. Energy Economics 16: 115-120.

Chen, Yen Yin, and Jung Hua Wu. 2008. Simple Keynesian input-output structural decomposition analysis using weighted Shapley value resolution. Annals of Regional Science 42: 879-892.

Dietzenbacher, Erick, and Bart Los. 1998. Structural decomposition techniques: sense and sensitivity. Economic Systems Research 10: 307-324.

Doan, Ha Thi Thanh, and Trinh Quang Long. 2019. Technical change, exports, and employment growth in china: A structural decomposition analysis. Asian Economic Papers 18: 29-46.

Eurostat 2015a. ESA Supply, use and input-output tables - Eurostat. Available online: https://ec.europa.eu/eurostat/web/esa-supplyuse-input-tables/data/database. (Accessed on 25 March 2020).

Eurostat 2015b. Data Explorer. Available online: https://appsso.eurostat.ec.europa.eu/nui/show.do?dataset=nama_10_a64\&lang=en. (Accessed on 25 March 2020).

Fang, Dan, and Jin Yang. 2021. Drivers and critical supply chain paths of black carbon emission: A structural path decomposition. Journal of Environmental Management 278: 111514.

Feng, Le, Bin Chen, Tasawar Hayat, Ahmed Alsaedi, and Bashir Ahmad. 2017. The driving force of water footprint under the rapid urbanization process: a structural decomposition analysis for Zhangye city in China. Journal of Cleaner Production 163: S322S328.

Forssell, Osmo. 1990. The input-output framework for analysing changes in the use of labour by education levels. Economic Systems Research 2: 363-376.

Fujimagari, David. 1989. The sources of change in canadian industry output. Economic Systems Research 1: 187-202.

Gerveni, Maria, Andre F T Avelino, and Sandy Dall'erba. 2020. Drivers of Water Use in the Agricultural Sector of the European Union 27. Environmental Science and Technology 54: 9191-9199.

Gim, Ho Un, and Koonchan Kim. 2005. The decomposition by factors in direct and indirect requirements: with applications to estimating the pollution generation. The Korean Economic Review 21: 309-325.

Gim, Ho Un, and Koonchan Kim. 1998. The general relation between two different notions of direct and indirect input requirements. Journal of Macroeconomics 20: 199-208.

Han, Xiaoli. 1995. Structural change and labor requirement of the Japanese economy. Economic Systems Research 7: 47-66.

Han, Xiaoli, and T. K. Lakshmanan. 1994. Structural changes and energy consumption in the Japanese economy 1975-85: An inputoutput analysis. Energy Journal 15: 165-188.

Hoekstra, Rutger, and J C J M Van Der Bergh. 2003. Comparing structural and index decomposition analysis. Energy Economics 25: 39-64.

Jacobsen, H. K. 2000. Energy demand, structural change and trade: A decomposition analysis of the Danish manufacturing industry. Economic Systems Research 12: 319-343.

Jeong, Ki Jun. 1982. Direct and indirect requirements: A correct economic interpretation of the Hawkins-Simon conditions. Journal of Macroeconomics 4: 349-356.

Jeong, Ki Jun. 1984. The relation between two different notions of direct and indirect input requirements. Journal of Macroeconomics 6: $473-476$.

Li, Dongrui, Yalin Lei, Li Li, and Lingna Liu. 2020a. Study on industrial selection of counterpart cooperation between Jilin province and Zhejiang province in China from the perspective of low carbon. Environmental Science and Pollution Research 27: 1666816676.

Li, Wei, Yuyan Huang, and Can Lu. 2020b. Exploring the driving force and mitigation contribution rate diversity considering new 
normal pattern as divisions for carbon emissions in Hebei province. Journal of Cleaner Production 243: 118559.

Li, Xin, Xiaoqiong He, Xiyu Luo, Xiandan Cui, and Minxi Wang. 2020c. Exploring the characteristics and drivers of indirect energy consumption of urban and rural households from a sectoral perspective. Greenhouse Gases: Science and Technology 10: 907-924.

Liu, Dunnan, Xiaodan Guo, and Bowen Xiao. 2019. What causes growth of global greenhouse gas emissions? Evidence from 40 countries. Science of the Total Environment 661: 750-766.

Liu, Li-Jing, and Qiao-Mei Liang. 2017. Changes to pollutants and carbon emission multipliers in China 2007-2012: An input-output structural decomposition analysis. Journal of Environmental Management 203: 76-86.

Miller, Ronald E, and Peter D Blair. 2009. Input-Output Analysis: Fundations and Extensions (2nd Ed.). Cambridge University Press, Cambridge.

Mukhopadhyay, K., and D. Chakraborty. 1999. India's energy consumption changes during 1973/74 to 1991/92. Economic Systems Research 11: 423-438.

Qian, Yiying, Huijuan Dong, Yong Geng, Shaozhuo Zhong, Xu Tian, Yanhong Yu, Yihui Chen, and Dana Avery Moss. 2018. Water footprint characteristic of less developed water-rich regions: Case of Yunnan, China. Water Research 141: 208-216.

Rose, A., and S. Casler. 1996. Input-output structural decomposition analysis: a critical appraisal. Economic Systems Research 8: 33-62.

Rose, A., and C Y Chen. 1991. Sources of change in energy use in the US economy, 1972-1982: a structural decomposition analysis. Resources and Energy 13: 1-21.

Song, Yi, Jianbai Huang, Yijun Zhang, and Zhiping Wang. 2019. Drivers of metal consumption in China: An input-output structural decomposition analysis. Resources Policy 63: 101421.

Sonis, M., G. J.D. Hewings, and J. Guo. 1996. Sources of structural change in input-output systems: a field of influence approach. Economic Systems Research 8: 15-32.

Supasa, Tharinya, Shu-San Hsiau, Shih-Mo Lin, Wongkot Wongsapai, and Jiunn-Chi Wu. 2017. Household energy consumption behaviour for different demographic regions in Thailand from 2000 to 2010. Sustainability 9: 2328.

Wang, Fei, Baomin Dong, Xiaopeng Yin, and Chi An. 2014. China's structural change: A new SDA model. Economic Modelling 43: 256-266.

Wang, Xiaomeng, Kai Huang, Yajuan Yu, Tingting Hu, and Yanjie Xu. 2016. An input-output structural decomposition analysis of changes in sectoral water footprint in China. Ecological Indicators 69: 26-34.

Xu, Shichun, Wenwen Zhang, Qinbin Li, Bin Zhao, Shuxiao Wang, and Ruyin Long. 2017. Decomposition analysis of the factors that influence energy related air pollutant emission changes in China using the SDA method. Sustainability 9: 1742.

Yu, Yang, Tangyang Jiang, Shuangqi Li, Xiaolong Li, and Dingchao Gao. 2020. Energy-related CO2 emissions and structural emissions' reduction in China's agriculture: An input-output perspective. Journal of Cleaner Production 276: 124169.

Zhi, Y., Z. F. Yang, and X. A. Yin. 2014. Decomposition analysis of water footprint changes in a water-limited river basin: A case study of the Haihe River basin, China. Hydrology and Earth System Sciences 18: 1549-1559. 